\title{
RAPID SOLUTION OF MINIMAL RIESZ ENERGY PROBLEMS
}

\author{
H. HARBRECHT, W.L. WENDLAND, AND N. ZORII
}

\begin{abstract}
In $\mathbb{R}^{n}, n \geq 2$, we compute the solution to both the unconstrained and constrained Gauss variational problem, considered for the Riesz kernel $\|\mathbf{x}-\mathbf{y}\|^{\alpha-n}$ of order $1<\alpha<n$ and a pair of compact, disjoint, boundaryless $(n-1)$ dimensional $C^{k-1,1}$-manifolds $\Gamma_{i}, i=1,2$, where $k>(\alpha-1) / 2$, each $\Gamma_{i}$ being charged with Borel measures with the sign $\alpha_{i}:= \pm 1$ prescribed. Such variational problems over a cone of Borel measures can be formulated as minimization problems over the corresponding cone of surface distributions belonging to the SobolevSlobodetski space $H^{-\varepsilon / 2}(\Gamma)$, where $\varepsilon:=\alpha-1$ and $\Gamma:=\Gamma_{1} \cup \Gamma_{2}$ (see H. Harbrecht, W.L. Wendland, and N. Zorii. [Math. Nachr. 287 (2014) 48-69]). We thus approximate the sought density by piecewise constant boundary elements and apply the primal-dual active set strategy to impose the desired inequality constraints. The boundary integral operator which is defined by the Riesz kernel under consideration is efficiently approximated by means of an $\mathcal{H}$-matrix approximation. This particularly enables the application of a preconditioner for the iterative solution of the first order optimality system. Numerical results in $\mathbb{R}^{3}$ are given to demonstrate our approach.
\end{abstract}

\section{INTRODUCTION}

The present article is devoted to the numerical solution of minimal Riesz energy problems in $\mathbb{R}^{n}, n \geq 2$. Problems of this kind go back to Carl Friedrich Gauss who investigated in [10] the variational problem of minimizing the Newtonian energy evaluated in the presence of an external field, nowadays called the Gauss functional, over a nonnegative charge distribution $\varphi \mathrm{d} s$ on the boundary surface of a given bounded domain. We minimize the Gauss functional for both the unconstrained and constrained problem (see Section 2 for the strict definitions), considered for the Riesz kernel $\|\mathbf{x}-\mathbf{y}\|^{\alpha-n}$ of order $1<\alpha<n$ and a pair of compact, disjoint, boundaryless $(n-1)$-dimensional $C^{k-1,1}$-manifolds $\Gamma_{i}, i=1,2$, where $k>(\alpha-1) / 2$, each of which being charged with Borel measures with the sign +1 and -1 , respectively. Notice that $\Gamma_{2}=\varnothing$ is explicitly allowed.

Key words and phrases. Gauss variational problem, Riesz kernel, boundary element method, active set strategy. 
In applications, the numerical solution of both the unconstrained and constrained Gauss variational problem is of great interest. Particularly, in electrical engineering, capacitors are modeled in this way where for practical reasons on each $\Gamma_{i}, i=$ 1,2 , either a nonnegative or a nonpositive charge is allowed (see "capacitors" in [26]). The discrete counterpart of the Gauss variational problem is related to the classical Thomson problem which concerns finding the ground state of $N$ equally charged particles on the sphere in a Coulomb potential field (see [36] and [1] for generalizations to arbitrary surfaces). The Gauss variational problem has also impact on approximation theory and the development of efficient quadrature rules (see [19]).

The unconstrained Gauss variational problem is that of minimizing the Gauss functional over an affine cone of all (signed) Borel measures $\nu=\nu^{1}-\nu^{2}$ of finite Riesz energy, where $\nu^{i}, i=1,2$, is nonnegative, supported by $\Gamma_{i}$, and satisfies some normalizing assumption for $i=1,2$. As has been proved in [18, Theorems 5.1, 7.1], under some additional assumptions, this problem can be reformulated as a minimization problem over the corresponding cone of surface distributions belonging to the Sobolev-Slobodetski space $H^{-\varepsilon / 2}(\Gamma)$, where $\varepsilon:=\alpha-1$ and $\Gamma:=\Gamma_{1} \cup \Gamma_{2}$. It is clear that a corresponding equivalent formulation is also valid if, in addition to the aforementioned requirements, the admissible measures on $\Gamma_{1}$ and $\Gamma_{2}$ are constrained from above as in $[39,40,42,43]$ (exactly such a problem is called constrained). Thus, problems of these kinds can be solved numerically by employing fast boundary integral equation methods in combination with a projected gradient method to compute the solution. This approach has been proposed and studied in a series of articles $[17,18,30]$ which are concerned with the development of efficient solution techniques for the unconstrained Gauss variational problem relative to the Riesz (in particular, Newtonian) kernel.

Unfortunately, as observed in $[17,30]$, the convergence of the projected gradient method is extremely slow. Indeed, it constitutes the bottleneck of the existing numerical method. In the present article, we therefore employ the primal-dual active set strategy for the solution of the aforementioned minimization problems. It can be reinterpreted as a semi-smooth Newton method and converges thus superlinearly, see $[6,22,24,28]$ and references cited therein. In particular, this enables us to treat the constrained problem as well. In this case the density is not only nonnegative, but also less than a given upper threshold (compare with $[17,18,30]$ ). By numerical examples we demonstrate that the active set strategy indeed drastically reduces the number of iterations which are required to compute the minimizing density.

The rest of this article is organized as follows. In Section 2, we precisely state the unconstrained and constrained Gauss variational problem relative to the Riesz 
kernel. Then, in Section 3, the sought density is discretized by piecewise constant ansatz functions. The particular active set strategy for the iterative solution of the optimization problem is introduced in Section 4. Numerical results are presented in Section 5 for a single boundary manifold, as well as for two boundary manifolds immersed into $\mathbb{R}^{3}$. Finally, in Section 6, we state concluding remarks.

\section{UnCONSTRAined AND CONSTRAINED GAUss VARIATIONAL PROBLEMS}

In $\mathbb{R}^{n}, n \geq 2$, consider the Riesz kernel $\|\mathbf{x}-\mathbf{y}\|^{\alpha-n}$ of order $0<\alpha<n$ and an ordered pair $\mathbf{A}$ of nonempty, compact and disjoint sets $A_{1}$ and $A_{2}$ with nonzero Riesz capacity (For the definition of capacity, see, e.g., [29]). Having fixed a numerical vector $\mathbf{a}:=\left(a_{1}, a_{2}\right)$ with $a_{i}>0, i=1,2$, and a positive, continuous function $g$ on $A:=A_{1} \cup A_{2}$, we define $\mathcal{E}_{\alpha}(\mathbf{A}, \mathbf{a}, g)$ as the class of all (signed) Borel measures $\nu=\nu^{1}-\nu^{2}$ with finite Riesz energy

$$
I_{\alpha}(\nu):=\int\|\mathbf{x}-\mathbf{y}\|^{\alpha-n} \mathrm{~d}(\nu \otimes \nu)(\mathbf{x}, \mathbf{y})
$$

such that $\nu^{i}$ is nonnegative, supported on $A_{i}$ and satisfies the normalizing assumptions $\int g \mathrm{~d} \nu^{i}=a_{i}$ for $i=1,2$. If, moreover, $f$ is a continuous external field, then the (unconstrained) Gauss variational problem is that of minimizing the Gauss functional

$$
G_{f}(\nu):=I_{\alpha}(\nu)+2 \int f \mathrm{~d} \nu
$$

where $\nu$ ranges over the class $\mathcal{E}_{\alpha}(\mathbf{A}, \mathbf{a}, g)$.

For more theoretical background to the unconstrained Gauss variational problem, we refer the reader to $[31,37,38,39,41,44] .{ }^{1}$ In particular, it has been shown therein that, in the case under consideration, a solution to such a problem exists. This follows easily from the fact that, for compact and disjoint $A_{i}, i=1,2$, and continuous $g$ and $f$, the class $\mathcal{E}_{\alpha}(\mathbf{A}, \mathbf{a}, g)$ is weakly compact, while the Gauss functional $G_{f}(\nu)$ is weakly lower semicontinuous. However, in general, this is no longer true if any of the $A_{i}$ would be noncompact (see, e.g., [37, Theorem 2]). Necessary and sufficient conditions for the Gauss variational problem to be, nevertheless, solvable, though some of the conductors are noncompact, can be found in [41]. There, a finer approach based on the pre-Hilbert structure in the space of all (signed) measures with finite Riesz energy and some completeness results has been developed and efficiently employed. Furthermore, a solution to the Gauss variational problem is unique (provided it exists), which follows from the convexity of the class $\mathcal{E}_{\alpha}(\mathbf{A}, \mathbf{a}, g)$ and the strict positive definiteness of the Riesz kernel (see, e.g., [39, Lemma 6]).

\footnotetext{
${ }^{1}$ For the logarithmic kernel in the plane, see also [34] and the references given therein.
} 
Fix, in addition, a measure $\omega=\omega^{1}-\omega^{2}$ with finite Riesz energy such that $\omega^{i}$, $i=1,2$, are nonnegative and supported by $A_{i}, i=1,2$, respectively, and define

$$
\mathcal{E}_{\alpha}^{\omega}(\mathbf{A}, \mathbf{a}, g):=\left\{\nu \in \mathcal{E}_{\alpha}(\mathbf{A}, \mathbf{a}, g): \nu^{i} \leqslant \omega^{i} \text { for all } i=1,2\right\}
$$

where $\nu^{i} \leqslant \omega^{i}$ means that $\omega^{i}-\nu^{i} \geq 0$. The problem of minimizing the Gauss functional $G_{f}(\nu)$, where $\nu$ ranges over the class $\mathcal{E}_{\alpha}^{\omega}(\mathbf{A}, \mathbf{a}, g)$, is called the constrained Gauss variational problem (see, e.g., [39, 40, 42, 43]; see also [9, 33] where the case of the logarithmic kernel in the plane has been investigated).

Similar as before (i.e., as for the unconstrained Gauss variational problem), a solution to the constrained problem exists because $\mathcal{E}_{\alpha}^{\omega}(\mathbf{A}, \mathbf{a}, g)$ remains to be weakly compact. Again, in general, this is no longer true if any of the $A_{i}$ would be noncompact (see, e.g., [40, Theorem 2]). Necessary and sufficient conditions for the constrained problem to be, nevertheless, solvable, though some of the conductors are noncompact, are given in [40, Theorem 3]. Furthermore, a solution to the constrained problem is unique (provided that it exists), which is shown using the same arguments as in the case of the unconstrained problem (see, e.g., [39, Lemma 6]).

In all that follows, we assume that $1<\alpha<n$ and $A_{i}=\Gamma_{i}, i=1,2$, where $\Gamma_{i}$ is a compact, boundaryless $(n-1)$-dimensional $C^{k-1,1}$-manifold with $k>(\alpha-1) / 2$. Write $\Gamma:=\Gamma_{1} \cup \Gamma_{2}$ and $\varepsilon:=\alpha-1$. Then, one can define the boundary integral operator

$$
(V \lambda)(\mathbf{x}):=\int_{\Gamma} \frac{\lambda(\mathbf{y})}{\|\mathbf{x}-\mathbf{y}\|^{n-\alpha}} \mathrm{d} \sigma(\mathbf{y}), \quad \text { where } \mathbf{x} \in \Gamma \text { and } \lambda \in H^{-\varepsilon / 2}(\Gamma)
$$

which maps $H^{-\varepsilon / 2}(\Gamma)$ bijectively onto $H^{\varepsilon / 2}(\Gamma)$ (see [18, Theorem 4.3]). Notice that, for our purposes, it is enough to assume $\Gamma_{i}, i=1,2$, to be Lipschitz, provided that $n=2$ or $n=3$. But, if $n \geq 4$, the case $\alpha \geq 3$ may appear and then more regularity than Lipschitz is required.

In accordance with $[17,18,30]$, for $f, g \in H^{\varepsilon / 2}(\Gamma)$, the Gauss variational problem on $\Gamma$ has the following equivalent formulation. Given a continuous external field $f \in H^{\varepsilon / 2}(\Gamma)$ and a continuous nonnegative weight function $g \in H^{\varepsilon / 2}(\Gamma)$, find the minimizer of the quadratic functional

$$
E(\lambda)=(V \lambda, \lambda)_{L^{2}(\Gamma)}+2(f, \lambda)_{L^{2}(\Gamma)},
$$

where $\lambda$ ranges over the subset of $H^{-\varepsilon / 2}(\Gamma)$ with the additional properties that

$$
\begin{aligned}
& \left.\lambda\right|_{\Gamma_{1}} \geq 0, \quad(\lambda, g)_{L^{2}\left(\Gamma_{1}\right)}=a_{1}>0 \\
& \left.\lambda\right|_{\Gamma_{2}} \leq 0, \quad-(\lambda, g)_{L^{2}\left(\Gamma_{2}\right)}=a_{2}>0
\end{aligned}
$$


(Recall, that $a_{1}$ and $a_{2}$ are the weighted total charges on $\Gamma_{1}$ and $\Gamma_{2}$, respectively.) Observe that, under the stated assumptions on $\alpha, g, f$, and $\Gamma$, such a problem is uniquely solvable (see [18, Theorem 6.1]).

In addition to the unconstrained Gauss variational problem, we will also consider the constrained problem which is obtained by additionally imposing the upper constraint

$$
|\lambda| \leq h \quad \text { on } \Gamma
$$

Here, $h \in H^{-\varepsilon / 2}(\Gamma)$ is a nonnegative function such that $(h, g)_{L^{2}\left(\Gamma_{i}\right)} \geq a_{i}, i=1,2$. Notice that, under the stated assumptions on $\alpha, g, f, h$, and $\Gamma$, such a problem is uniquely solvable. This follows from the aforementioned results from [39, 40, 42, 43] with the help of the same arguments as in [18] (see Theorems 5.1 and 6.1 therein).

\section{GALERKin DiscRetization}

3.1. Surface representation. In the following, we focus on the particular situation that $\Gamma=\Gamma_{1} \cup \Gamma_{2}$ is a Lipschitz two-dimensional manifold immersed into $\mathbb{R}^{n}, n=3$. Nevertheless, all definitions and algorithms can be generalized to $n=2$ as well as to $n \geq 4$ in a straightforward way, provided that $\Gamma_{i}, i=1,2$, are $C^{k-1,1}$-manifolds, where $k>(\alpha-1) / 2$.

We shall numerically solve the constrained Gauss variational problem (2.2)-(2.4) by means of the Galerkin scheme. To this end, we shall assume that the manifold $\Gamma$ is given as a parametric surface consisting of smooth four-sided patches. Let $\square:=[0,1]^{2}$ denote the unit square. We subdivide the given manifold into several patches

$$
\Gamma=\bigcup_{k=1}^{M} \Gamma^{(k)}, \quad \Gamma^{(k)}=\gamma^{(k)}(\square), \quad k=1,2, \ldots, M
$$

such that each $\gamma^{(k)}: \square \rightarrow \Gamma^{(k)}$ defines a diffeomorphism of $\square$ onto $\Gamma^{(k)}$. The intersection $\Gamma^{(k)} \cap \Gamma^{(\ell)}, k \neq \ell$, of the patches $\Gamma^{(k)}$ and $\Gamma^{(\ell)}$ is supposed to be either empty, a common edge, or a common vertex.

With this surface representation at hand, it is easily possible to generate a nested sequence of meshes on the surface $\Gamma$. A mesh $\mathcal{T}_{j}$ on level $j$ for $\Gamma$ is induced by dyadic subdivisions of depth $j$ of the unit square into $4^{j}$ congruent squares, each of which is lifted to $\Gamma$ by the associated parameterization $\gamma^{(k)}$ (see Figure 3.1 for a visualization). We will refer to the particular elements as $\Gamma^{(k, j, \ell)}$ where $k$ is the index of the applied parameterization $\gamma^{(k)}, j$ is the level of the element and $\ell$ is the index of the element. Notice that the meshes $\mathcal{T}_{j}=\left\{\Gamma^{(k, j, \ell)}\right\}_{k, \ell}$ form regular meshes of $\Gamma$, provided that the parametric representation is globally continuous. 


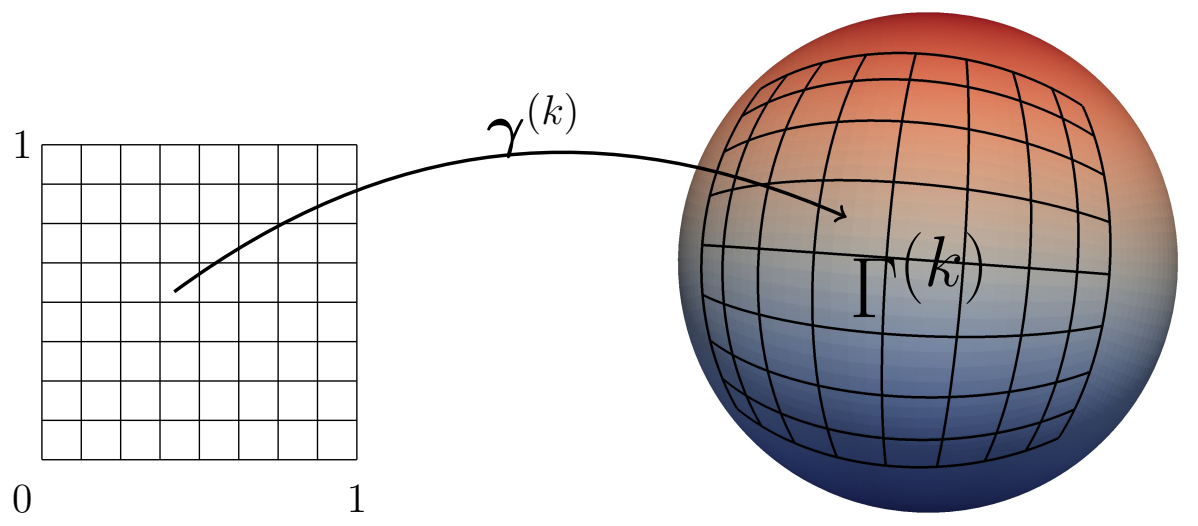

FiguRE 3.1. Surface representation and mesh generation.

The given surface representation differs from the usual approximation of surfaces by panels but has the advantage that we intrinsically arrive at a multilevel discretization. Technical surfaces generated by tools from Computer Aided Design (CAD) are often represented in the present form. There are several realizations of the parameterizations including B-splines, NURBS (nonuniform rational B-Splines), surfaces of revolution, and tabulated cylinders [23].

3.2. Boundary elements. Given the mesh $\mathcal{T}_{j}$, we shall consider the space $\mathcal{S}_{j}(\Gamma)=$ $\mathcal{S}_{j}\left(\Gamma_{1}\right) \oplus \mathcal{S}_{j}\left(\Gamma_{2}\right)$ of piecewise constant functions as the trial space and as the test space. By construction, these ansatz spaces are nested, i.e.,

$$
\mathcal{S}_{0}(\Gamma) \subset \mathcal{S}_{1}(\Gamma) \subset \cdots \subset \mathcal{S}_{j}(\Gamma) \subset \cdots \subset H^{-\varepsilon / 2}(\Gamma)
$$

The number of degrees of freedom of the space $\mathcal{S}_{j}\left(\Gamma_{i}\right)$ on $\Gamma_{i}$ is denoted by $m_{i}$ (i.e., $\left.\operatorname{dim} \mathcal{S}_{j}\left(\Gamma_{i}\right)=m_{i}\right), i=1,2$. Notice that $m_{1} \sim m_{2} \sim 4^{j}$ by construction. Moreover, we call the (row) vector of the related $L^{2}$-normalized piecewise constant basis functions by $\Phi_{1}$ and $\Phi_{2}$, respectively. This means that any piecewise constant function $\lambda_{i} \in$ $\mathcal{S}_{j}\left(\Gamma_{i}\right)$ can be written as $\lambda_{i}=\Phi_{i} \boldsymbol{\lambda}_{i}$ with a certain coefficient (column) vector $\boldsymbol{\lambda}_{i}=$ $\left(\lambda_{i, k}\right)_{k} \in \mathbb{R}^{m_{i}}$

3.3. Discrete minimization problem. Define the data vectors

$$
\mathbf{f}_{i}:=\left(f, \Phi_{i}\right)_{L^{2}\left(\Gamma_{i}\right)}, \quad \mathbf{g}_{i}:=\frac{1}{a_{i}}\left(g, \Phi_{i}\right)_{L^{2}\left(\Gamma_{i}\right)}, \quad \mathbf{h}_{i}:=\left(h, \Phi_{i}\right)_{L^{2}\left(\Gamma_{i}\right)}, \quad i=1,2,
$$

and the system matrices

$$
\mathbf{V}_{i, j}:=\left(V \Phi_{j}, \Phi_{i}\right)_{L^{2}\left(\Gamma_{i}\right)}, \quad i, j=1,2
$$


Then, the Galerkin formulation of the constrained minimization problem (2.2)-(2.4) reads as follows. Find $\lambda=\Phi_{1} \boldsymbol{\lambda}_{1}-\Phi_{2} \boldsymbol{\lambda}_{2} \in \mathcal{S}_{j}(\Gamma) \subset H^{-\varepsilon / 2}(\Gamma)$ such that

$$
E\left(\lambda_{1}-\lambda_{2}\right):=\left[\begin{array}{c}
\boldsymbol{\lambda}_{1} \\
\boldsymbol{\lambda}_{2}
\end{array}\right]^{\top}\left[\begin{array}{cc}
\mathbf{V}_{1,1} & -\mathbf{V}_{1,2} \\
-\mathbf{V}_{2,1} & \mathbf{V}_{2,2}
\end{array}\right]\left[\begin{array}{l}
\boldsymbol{\lambda}_{1} \\
\boldsymbol{\lambda}_{2}
\end{array}\right]+2\left[\begin{array}{l}
\mathbf{f}_{1} \\
\mathbf{f}_{2}
\end{array}\right]^{\top}\left[\begin{array}{l}
\boldsymbol{\lambda}_{1} \\
\boldsymbol{\lambda}_{2}
\end{array}\right] \rightarrow \min
$$

subject to

$$
\mathbf{g}_{i}^{\top} \boldsymbol{\lambda}_{i}=1, \quad \mathbf{0} \leq \boldsymbol{\lambda}_{i} \leq \mathbf{h}_{i}, \quad i=1,2 .
$$

This approximation is a conforming method where the trial and test spaces belong to the energy space $H^{-\varepsilon / 2}(\Gamma)$. It is conceptionally different from the method in [2] which is a nonconforming method based on spatial Dirac distributions $\delta_{\mathbf{x}}(\mathbf{y})$. If $\mathbf{x} \in \Gamma$, then $\delta_{\mathbf{x}}(\mathbf{y})$ does not have a finite Riesz energy, cf. [29, Chapter II, $\left.\S 3\right]$. On the discrete level with a finite number of Dirac distributions $\delta_{\mathbf{x}_{i}}(\mathbf{y}), \mathbf{x}_{i} \in \Gamma$, however, the discrete energy becomes finite if the diagonal terms are omitted.

Problem (3.6) is a quadratic minimization problem with linear equality and inequality constraints (3.7). In particular, it is a convex problem since the system matrix $\mathbf{V}=\left[\begin{array}{cc}\mathbf{V}_{1,1} & -\mathbf{V}_{1,2} \\ -\mathbf{V}_{2,1} & \mathbf{V}_{2,2}\end{array}\right]$ is positive definite. Moreover, note that the inequality constraints in (3.7) are standard box constraints.

In $[17,18,30]$, we solved the unconstrained Gauss variational problem (i.e., problem (3.6) and (3.7) without the upper constraints $\boldsymbol{\lambda}_{i} \leq \mathbf{h}_{i}, i=1,2$ ), by imposing the equality constraint in (3.7) using a penalty term for the defect and then applying a projected gradient scheme. That is, we minimized the energy functional

$$
E_{\rho}\left(\lambda_{1}-\lambda_{2}\right):=E\left(\lambda_{1}-\lambda_{2}\right)+\frac{\rho}{2}\left(\mathbf{g}_{1}^{\top} \boldsymbol{\lambda}_{1}-1\right)^{2}+\frac{\rho}{2}\left(\mathbf{g}_{2}^{\top} \boldsymbol{\lambda}_{2}-1\right)^{2} \rightarrow \min
$$

subject to the constraints $\mathbf{0} \leq \boldsymbol{\lambda}_{i}, i=1,2$. The linear speed of convergence of this iterative method has been proven in $[17,30]$, which, however, depends strongly on the degrees of freedom and turned out to be extremely slow. Therefore, we shall propose an alternative numerical approach in Section 4.

3.4. First order optimality conditions. For $i=1,2$, we shall introduce Lagrange multipliers $\kappa_{i} \in \mathbb{R}$ and $\boldsymbol{\mu}_{i}=\left[\mu_{i, j}\right], \boldsymbol{\nu}_{i}=\left[\nu_{i, j}\right] \in \mathbb{R}^{m_{i}}$. Then, the Lagrangian for the discrete minimization problem (3.6) and (3.7) reads as follows:

$$
\begin{gathered}
F\left(\boldsymbol{\lambda}_{1}, \boldsymbol{\lambda}_{2}, \kappa_{1}, \kappa_{2}, \boldsymbol{\mu}_{1}, \boldsymbol{\mu}_{2}, \boldsymbol{\nu}_{1}, \boldsymbol{\nu}_{2}\right):=\left[\begin{array}{c}
\boldsymbol{\lambda}_{1} \\
\boldsymbol{\lambda}_{2}
\end{array}\right]^{\top}\left[\begin{array}{cc}
\mathbf{V}_{1,1} & -\mathbf{V}_{1,2} \\
-\mathbf{V}_{2,1} & \mathbf{V}_{2,2}
\end{array}\right]\left[\begin{array}{c}
\boldsymbol{\lambda}_{1} \\
\boldsymbol{\lambda}_{2}
\end{array}\right] \\
+\left[\begin{array}{c}
\kappa_{1} \\
\kappa_{2}
\end{array}\right]^{\top}\left[\begin{array}{l}
\mathbf{g}_{1}^{\top} \boldsymbol{\lambda}_{1}-1 \\
\mathbf{g}_{2}^{\top} \boldsymbol{\lambda}_{2}-1
\end{array}\right]-\left[\begin{array}{c}
\boldsymbol{\mu}_{1} \\
\boldsymbol{\mu}_{2}
\end{array}\right]^{\top}\left[\begin{array}{c}
\boldsymbol{\lambda}_{1} \\
\boldsymbol{\lambda}_{2}
\end{array}\right]+\left[\begin{array}{c}
\boldsymbol{\nu}_{1} \\
\boldsymbol{\nu}_{2}
\end{array}\right]^{\top}\left[\begin{array}{l}
\boldsymbol{\lambda}_{1}-\mathbf{h}_{1} \\
\boldsymbol{\lambda}_{2}-\mathbf{h}_{2}
\end{array}\right]
\end{gathered}
$$


Hence, according to the Karush-Kuhn-Tucker (KKT) theorem (see [25, 27]), the first order necessary conditions for the optimal solution $\left(\boldsymbol{\lambda}_{1}^{\star}, \boldsymbol{\lambda}_{2}^{\star}\right)$ of the discrete minimization problem read as follows: there exist Lagrange multipliers $\left(\kappa_{1}^{\star}, \kappa_{2}^{\star}, \boldsymbol{\mu}_{1}^{\star}, \boldsymbol{\mu}_{2}^{\star}, \nu_{1}^{\star}, \boldsymbol{\nu}_{2}^{\star}\right)$ such that

$$
\begin{gathered}
{\left[\begin{array}{cc}
\mathbf{V}_{1,1} & -\mathbf{V}_{1,2} \\
-\mathbf{V}_{2,1} & \mathbf{V}_{2,2}
\end{array}\right]\left[\begin{array}{c}
\boldsymbol{\lambda}_{1}^{\star} \\
\boldsymbol{\lambda}_{2}^{\star}
\end{array}\right]+\left[\begin{array}{c}
\kappa_{1}^{\star} \mathbf{g}_{1} \\
\kappa_{2}^{\star} \mathbf{g}_{2}
\end{array}\right]-\left[\begin{array}{c}
\boldsymbol{\mu}_{1}^{\star} \\
\boldsymbol{\mu}_{2}^{\star}
\end{array}\right]+\left[\begin{array}{c}
\boldsymbol{\nu}_{1}^{\star} \\
\boldsymbol{\nu}_{2}^{\star}
\end{array}\right]=\mathbf{0},} \\
\kappa_{1}^{\star}\left(\mathbf{g}_{1}^{\top} \boldsymbol{\lambda}_{1}^{\star}-1\right)=0, \quad \kappa_{2}^{\star}\left(\mathbf{g}_{2}^{\top} \boldsymbol{\lambda}_{2}^{\star}-1\right)=0 \\
\boldsymbol{\mu}_{1}^{\top} \boldsymbol{\lambda}_{1}^{\star}=0, \quad \boldsymbol{\mu}_{2}^{\top} \boldsymbol{\lambda}_{2}^{\star}=0 \\
\boldsymbol{\mu}_{1}^{\top}\left(\boldsymbol{\lambda}_{1}^{\star}-\mathbf{h}_{1}\right)=0, \quad \boldsymbol{\mu}_{2}^{\top}\left(\boldsymbol{\lambda}_{2}^{\star}-\mathbf{h}_{2}\right)=0
\end{gathered}
$$

together with the primal feasibility

$$
\mathbf{g}_{1}^{\top} \boldsymbol{\lambda}_{1}^{\star}=1, \quad \mathbf{g}_{2}^{\top} \boldsymbol{\lambda}_{2}^{\star}=1, \quad \mathbf{0} \leq \boldsymbol{\lambda}_{1}^{\star} \leq \mathbf{h}_{1}, \quad \mathbf{0} \leq \boldsymbol{\lambda}_{2}^{\star} \leq \mathbf{h}_{2}
$$

and the dual feasibility

$$
\mu_{1}^{\star} \geq 0, \quad \mu_{2}^{\star} \geq 0, \quad \nu_{1}^{\star} \geq 0, \quad \nu_{2}^{\star} \geq 0
$$

Notice that the relation " $\geq$ " applied to vectors has to be understood component by component.

\section{Iterative SOLUTiON}

4.1. Primal-dual active set strategy. For the numerical solution of the constrained discrete optimization problem (3.6) and (3.7), we apply the primal-dual active set strategy. This is an iterative method for solving minimization problems with inequality constraints and has been introduced in [6]. It replaces successively the inequality constraints by the related equality constraints for all the indices where the constraint becomes active.

Given an iterate $\left(\boldsymbol{\lambda}_{1}^{(\ell-1)}, \boldsymbol{\lambda}_{2}^{(\ell-1)}, \kappa_{1}^{(\ell-1)}, \kappa_{2}^{(\ell-1)}, \boldsymbol{\mu}_{1}^{(\ell-1)}, \boldsymbol{\mu}_{2}^{(\ell-1)}, \boldsymbol{\nu}_{1}^{(\ell-1)}, \boldsymbol{\nu}_{2}^{(\ell-1)}\right)$, we stop the algorithm if

$$
\begin{gathered}
\left\|\left[\begin{array}{cc}
\mathbf{V}_{1,1} & -\mathbf{V}_{1,2} \\
-\mathbf{V}_{2,1} & \mathbf{V}_{2,2}
\end{array}\right]\left[\begin{array}{c}
\boldsymbol{\lambda}_{1}^{(\ell)} \\
\boldsymbol{\lambda}_{2}^{(\ell-1)}
\end{array}\right]+\left[\begin{array}{c}
\kappa_{1}^{(\ell)} \mathbf{g}_{1} \\
\kappa_{2}^{(\ell-1)} \mathbf{g}_{2}
\end{array}\right]-\left[\begin{array}{c}
\boldsymbol{\mu}_{1}^{(\ell)} \\
\boldsymbol{\mu}_{2}^{(\ell-1)}
\end{array}\right]+\left[\begin{array}{c}
\boldsymbol{\nu}_{1}^{(\ell)} \\
\boldsymbol{\nu}_{2}^{(\ell-1)}
\end{array}\right]\right\|_{2} \leq \varepsilon \\
\left|\mathbf{g}_{i}^{\top} \boldsymbol{\lambda}_{i}^{(\ell)}-1\right| \leq \varepsilon,\left\|\min \left\{\mathbf{0}, \boldsymbol{\lambda}_{i}^{(\ell)}\right\}\right\|_{2} \leq \varepsilon,\left\|\max \left\{\mathbf{0},\left(\boldsymbol{\lambda}_{i}^{(\ell)}-\mathbf{h}_{i}\right)\right\}\right\|_{2} \leq \varepsilon, \quad i=1,2 .
\end{gathered}
$$


Furthermore, for $i=1,2$, we define the sets $\underline{\mathbb{I}}_{i}^{(\ell)}$ and $\overline{\mathbb{I}}_{i}^{(\ell)}$ of active indices and $\underline{\mathbb{J}}_{i}^{(\ell)}$ and $\overline{\mathbb{J}}_{i}^{(\ell)}$ of inactive indices in accordance with

$$
\begin{gathered}
\underline{\mathbb{I}}_{i}^{(\ell)}:=\left\{k=\left\{1,2, \ldots, m_{i}\right\}: \mu_{i, k}^{(\ell)}-c \lambda_{i, k}^{(\ell)}<0\right\}, \\
\overline{\mathbb{I}}_{i}^{(\ell)}:=\left\{k=\left\{1,2, \ldots, m_{i}\right\}: \nu_{i, k}^{(\ell)}+c\left(\lambda_{i, k}^{(\ell)}-h_{i, k}\right)>0\right\}, \\
\underline{\mathbb{J}}_{i}^{(\ell)}:=\left\{1,2, \ldots, m_{i}\right\} \backslash \underline{\mathbb{I}}_{i}^{(\ell)}, \quad \overline{\mathbb{J}}_{i}^{(\ell)}:=\left\{1,2, \ldots, m_{i}\right\} \backslash \overline{\mathbb{I}}_{i}^{(\ell)},
\end{gathered}
$$

where $c>0$ is an appropriately chosen parameter (we choose $c=10^{-4}$ in our experiments). The sets $\underline{I}_{i}^{(\ell)}$ contain the indices of all boundary elements associated with the manifold $\Gamma_{i}$ for which the lower box constraint becomes active. The sets $\overline{\mathbb{I}}_{i}^{(\ell)}$ contain the indices of all boundary elements associated with the manifold $\Gamma_{i}$ for which the upper box constraint becomes active. This means, taking into account (3.8), we have to solve the saddle point problem

$$
\left[\begin{array}{cc|cc|cc|cc}
\mathbf{V}_{1,1} & -\mathbf{V}_{1,2} & \mathbf{g}_{1} & \mathbf{0} & -\mathbf{I}_{\mathbb{I}_{1}^{(k)}} & \mathbf{0} & \mathbf{I}_{\mathbb{\mathbb { I }}_{1}^{(k)}} & \mathbf{0} \\
-\mathbf{V}_{2,1} & \mathbf{V}_{2,2} & \mathbf{0} & \mathbf{g}_{2} & \mathbf{0} & -\mathbf{I}_{\mathbb{I}_{2}^{(k)}} & \mathbf{0} & \mathbf{I}_{\overline{\mathbb{I}}_{2}^{(k)}} \\
\hline \mathbf{g}_{1}^{\top} & \mathbf{0} & 0 & 0 & \mathbf{0} & \mathbf{0} & \mathbf{0} & \mathbf{0} \\
\mathbf{0} & \mathbf{g}_{2}^{\top} & 0 & 0 & \mathbf{0} & \mathbf{0} & \mathbf{0} & \mathbf{0} \\
\hline-\mathbf{I}_{\mathbb{I}_{1}^{(\ell)}}^{\top} & \mathbf{0} & \mathbf{0} & \mathbf{0} & \mathbf{0} & \mathbf{0} & \mathbf{0} & \mathbf{0} \\
\mathbf{0} & -\mathbf{I}_{\mathbb{I}_{2}^{(\ell)}}^{\top} & \mathbf{0} & \mathbf{0} & \mathbf{0} & \mathbf{0} & \mathbf{0} & \mathbf{0} \\
\hline \mathbf{I}_{\mathbb{\mathbb { I }}_{1}^{(\ell)}}^{\top} & \mathbf{0} & \mathbf{0} & \mathbf{0} & \mathbf{0} & \mathbf{0} & \mathbf{0} & \mathbf{0} \\
\mathbf{0} & \mathbf{I}_{\overline{\mathbb{I}}_{2}^{(\ell)}}^{\top} & \mathbf{0} & \mathbf{0} & \mathbf{0} & \mathbf{0} & \mathbf{0} & \mathbf{0}
\end{array}\right]\left[\begin{array}{c}
\boldsymbol{\lambda}_{1}^{(\ell)+1} \\
\boldsymbol{\lambda}_{2}^{(\ell+1)} \\
\hline \kappa_{1}^{(\ell+1)} \\
\kappa_{2}^{(\ell+1)} \\
\hline \boldsymbol{\mu}_{1, \mathbb{I}_{1}^{(\ell)}}^{(\ell+1)} \\
\boldsymbol{\mu}_{2, \mathbb{I}_{2}^{(\ell)}}^{(\ell)} \\
\hline \boldsymbol{\nu}^{(\ell+1)} \\
1, \overline{\mathbb{I}}_{1}^{(\ell)} \\
\boldsymbol{\nu}_{2, \overline{\mathbb{I}}_{2}^{(\ell)}}^{(\ell)}
\end{array}\right]=\left[\begin{array}{c}
\mathbf{f}_{1} \\
\mathbf{f}_{2} \\
\hline 1 \\
1 \\
\mathbf{0} \\
\mathbf{0} \\
\hline \mathbf{h}_{1, \overline{\mathbb{I}}_{1}^{(\ell)}} \\
\mathbf{h}_{2, \overline{\mathbb{I}}_{2}^{(\ell)}}
\end{array}\right] .
$$

Here, the matrices $\mathbf{I}_{\mathbb{I}_{i}^{(\ell)}} \in \mathbb{R}^{m_{i} \times\left|\underline{I}_{i}^{(\ell)}\right|}$ and $\mathbf{I}_{\overline{\mathbb{I}}_{i}^{(\ell)}} \in \mathbb{R}^{m_{i} \times\left|\overline{\mathbb{I}}_{i}^{(\ell)}\right|}$ are obtained from the identity matrix in $\mathbb{R}^{m_{i}}$ by removing those columns whose indices are not contained in the index sets $\underline{\mathbb{I}}_{i}^{(\ell)}$ and $\overline{\mathbb{I}}_{i}^{(\ell)}$, respectively. Likewise, the vectors $\boldsymbol{\mu}_{i, \underline{I}_{i}^{(\ell)}}^{(\ell)} \in \mathbb{R}^{\left|\mathbb{I}_{i}^{(\ell)}\right|}$ and $\boldsymbol{\nu}_{i, \overline{\mathbb{I}}_{i}^{(\ell)}}^{(\ell)} \in$ $\mathbb{R}^{\left|\bar{\pi}_{i}^{(\ell)}\right|}$ consist only of those components of $\boldsymbol{\mu}_{i}^{(\ell)}$ and $\boldsymbol{\nu}_{i}^{(\ell)}$ which are contained in the index sets $\underline{\mathbb{I}}_{i}^{(\ell)}$ and $\overline{\mathbb{I}}_{i}^{(\ell)}$, respectively. The same holds true for $\mathbf{h}_{i, \overline{\mathbb{I}}_{i}^{(\ell)}} \in \mathbb{R}^{\left|\overline{\mathbb{I}}_{i}^{(\ell)}\right|}$.

For all inactive indices, the box constraints will be ignored and the associated components of the Lagrange multipliers are set to 0 :

$$
\mu_{i, k}^{(\ell+1)}=0 \text { for all } k \in \underline{\mathbb{J}}_{i}^{(\ell)}, \quad \nu_{i, k}^{(\ell+1)}=0 \text { for all } k \in \overline{\mathbb{J}}_{i}^{(\ell)}, \quad i=1,2 .
$$

Finally, the iteration index is increased $\ell \mapsto \ell+1$ and the loop restarted.

According to [22], the primal-dual active set strategy is equivalent to the semismooth Newton method which leads to the following result for its rate of convergence: 
Theorem 4.1. The iterates $\left(\boldsymbol{\lambda}_{1}^{(\ell)}, \boldsymbol{\lambda}_{2}^{(\ell)}\right)$ of the primal-dual active set strategy converge superlinearly to the optimal solution $\left(\boldsymbol{\lambda}_{1}^{\star}, \boldsymbol{\lambda}_{2}^{\star}\right)$ as $\ell \rightarrow \infty$, provided that the initial guess is appropriately chosen.

4.2. Multilevel iteration. The determination of the final active sets $\underline{I}_{i}^{\star}$ and $\overline{\mathbb{I}}_{i}^{\star}$ can be very costly. Hence, to enhance convergence, we suggest to exploit the fact that the trial spaces form a multilevel sequence; i.e., we suggest to exploit the fact that (3.5) holds.

Having determined the sets of active lower constraints and of active upper constraints on level $j-1$, the initial sets of active lower constraints and of active upper constraints on level $j$ are determined as follows. A coarse grid element whose index belongs to the set of active lower constraints on the coarse grid has four son elements on the fine grid. We add the respective indices to the set of active lower constraints $\underline{I}_{i}^{(0)}$. We proceed likewise with the sets of active upper constraints $\underline{I}_{i}^{(0)}$. This results in good initial guesses of the sets $\underline{\mathbb{I}}_{i}^{(0)}$ and $\overline{\mathbb{I}}_{i}^{(0)}$ of active indices on level $j$ and thus is a good initial guess $\left(\boldsymbol{\lambda}_{1}^{(0)}, \boldsymbol{\lambda}_{2}^{(0)}, \kappa_{1}^{(0)}, \kappa_{2}^{(0)}, \boldsymbol{\mu}_{1}^{(0)}, \boldsymbol{\mu}_{2}^{(0)}, \boldsymbol{\nu}_{1}^{(0)}, \boldsymbol{\nu}_{2}^{(0)}\right)$ for the primal-dual active set strategy by (4.9) and (4.10).

4.3. Rapid computation of the nonlocal operator. Since integral operators are nonlocal operators, the system matrix $\mathbf{V}$ is densely populated. This feature poses serious obstructions to the efficient numerical treatment of Riesz minimal energy problems. Fortunately, the system matrix is compressible in terms of an $\mathcal{H}$ matrix which drastically reduces the computational complexity, cf. [13]. To that end, we shall refer to the element $\Gamma^{\left(k^{\prime}, j^{\prime}, \ell^{\prime}\right)}$ also as a cluster. This means that we think of $\Gamma^{\left(k^{\prime}, j^{\prime}, \ell^{\prime}\right)}$ as the union $\left\{\Gamma^{(k, j, \ell)}: \Gamma^{(k, j, \ell)} \subset \Gamma^{\left(k^{\prime}, j^{\prime}, \ell^{\prime}\right)}\right\}$; i.e., the set of all tree leafs appended to $\Gamma^{\left(k^{\prime}, j^{\prime}, \ell^{\prime}\right)}$ or its sons. The mesh $\mathcal{T}_{j}$ induces therefore a collection of clusters which are ordered by a father-son relation, called the cluster tree, see Figure 4.2 for an illustration.

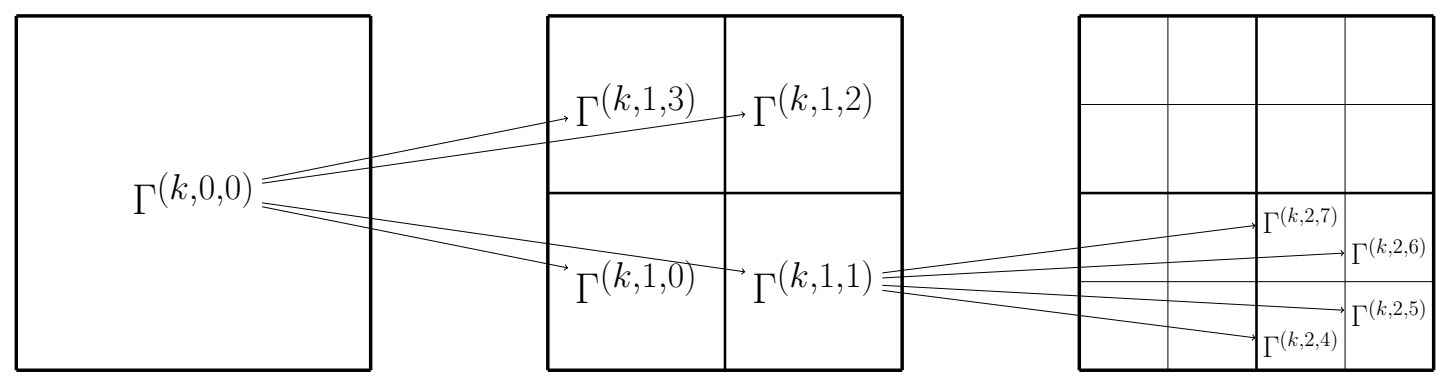

level 0 level 1 level 2

FiguRE 4.2. Visualization of the cluster tree. 
Definition 4.2. Two clusters $\Gamma^{\left(k, j^{\prime}, \ell\right)}$ and $\Gamma^{\left(k^{\prime}, j^{\prime}, \ell^{\prime}\right)}$ of the same level $j^{\prime} \leq j$ are called admissible if

$$
\max \left\{\operatorname{diam}\left(\Gamma^{\left(k, j^{\prime}, \ell\right)}\right), \operatorname{diam}\left(\Gamma^{\left(k^{\prime}, j^{\prime}, \ell^{\prime}\right)}\right)\right\} \leq \eta \operatorname{dist}\left(\Gamma^{\left(k, j^{\prime}, \ell\right)}, \Gamma^{\left(k^{\prime}, j^{\prime}, \ell^{\prime}\right)}\right)
$$

holds for some fixed $\eta \in(0,1)$. The collection of admissible blocks $\Gamma^{\left(k, j^{\prime}, \ell\right)} \times \Gamma^{\left(k^{\prime}, j^{\prime}, \ell^{\prime}\right)}$ forms the far-field of the operator. The remaining nonadmissible blocks correspond to the near-field of the operator.

By employing the admissibility condition, the quad-tree structure of the cluster tree $\mathcal{T}_{j}$ yields a block partitioning of the system matrix with quadratic blocks. In particular, each block on a particular level contains exactly the same number of element-element interactions, see also Figure 4.3 for a visualization of this special block partitioning of an $\mathcal{H}$-matrix. We refer the reader to [16] for further details.

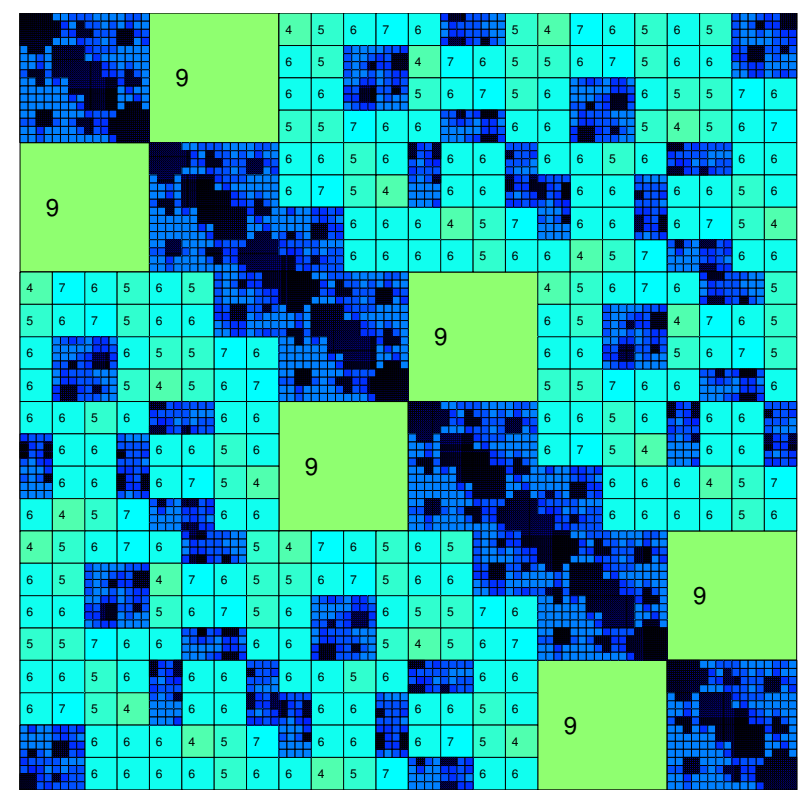

Figure 4.3. The block partitioning of the $\mathcal{H}$-matrix together with the approximation ranks.

The near-field operator has to be assembled in the traditional way while the far-field operator can be compressed as follows. Admissible matrix blocks can be compressed using two different approaches; namely, the Adaptive Cross Approximation (ACA) [3, 4] or the Fast Multipole Method (FMM) [12]. We apply here the first approach which approximates an admissible block $\widehat{\mathbf{V}}=\left(v_{i, j}\right)_{i, j=1}^{n} \in \mathbb{R}^{n \times n}$ using a truncated, partially pivoted Gaussian elimination. More precisely, we define vectors $\boldsymbol{\ell}_{m}, \mathbf{u}_{m} \in$ 
$\mathbb{R}^{n}$ by the following iterative scheme:

$$
\begin{aligned}
& \text { for } m=1,2, \ldots, \text { set } \mathbf{u}_{m}=\widehat{\mathbf{u}}_{m} / \widehat{u}_{m, j_{m}} \text { with } \\
& \qquad \widehat{\mathbf{u}}_{m}=\left(v_{i_{m}, j}\right)_{j=1}^{n}-\sum_{j=1}^{m-1} \ell_{j, i_{m}} \mathbf{u}_{j} \text { and } \boldsymbol{\ell}_{m}=\left(v_{i, j_{m}}\right)_{i=1}^{n}-\sum_{i=1}^{m-1} u_{i, j_{m}} \boldsymbol{\ell}_{i} .
\end{aligned}
$$

A criterion for guaranteeing the convergence of the algorithm is to choose the pivot element located in $\left(i_{m}, j_{m}\right)$-position as the maximum element in modulus of the remainder $\widehat{\mathbf{V}}-\mathbf{L}_{m-1} \mathbf{U}_{m-1}$, where we set $\mathbf{L}_{m-1}:=\left[\boldsymbol{\ell}_{1}, \ldots, \boldsymbol{\ell}_{m-1}\right]$ and $\mathbf{U}_{m-1}:=$ $\left[\mathbf{u}_{1}, \ldots, \mathbf{u}_{m-1}\right]^{\top}$. Since this would require assembling the whole matrix block $\widehat{\mathbf{V}}$, which is not feasible in practice, $j_{m}$ is chosen such that $\widehat{u}_{m, j_{m}}$ is the largest element in modulus of the row $\widehat{\mathbf{u}}_{m}$. Afterwards, the next index $i_{m+1}$ is chosen as the maximum element in modulus in the vector $\boldsymbol{\ell}_{m}$. We finally stop the iteration if the criterion

$$
\left\|\ell_{m+1}\right\|\left\|\mathbf{u}_{m+1}\right\| \leq \varepsilon\left\|\mathbf{L}_{m} \mathbf{U}_{m}\right\|_{F}
$$

holds for some desired accuracy $\varepsilon>0$, where $\|\cdot\|_{F}$ denotes the Frobenius norm.

The Riesz kernel under consideration is an asymptotically smooth kernel for all $\alpha<n$. Hence, as proven in [3] (see also [16] for the current realization on parametric surfaces), we have the following theorem for the adaptive cross approximation:

Theorem 4.3. Let $\mathbf{V}$ be the uncompressed system matrix and $\mathbf{V}_{\varepsilon}$ be the system matrix which is compressed by the adaptive cross approximation. Then, if the relative error per block with respect to the Frobenius norm is bounded by $\varepsilon$, there holds the error estimate

$$
\left\|\mathbf{V}-\mathbf{V}_{\varepsilon}\right\|_{F} \leq \varepsilon\|\mathbf{V}\|_{F}
$$

The cost-complexity to compute this approximation is of the order $\mathcal{O}\left(4^{j} j \log ^{2} \varepsilon\right)$.

4.4. Preconditioning. The boundary integral operator (2.1) acts between different Sobolev spaces which means that it acts on different length scales in a different way. It is well known that this causes the linear system to become more and more ill-conditioned when the level of resolution increases. Thus, preconditioning of the saddle-point problem (4.9) becomes an important issue when it is solved by an iterative solver (we use the MINRES method in our realization, cf. [11]). Following, e.g. $[5,32,35,45]$, we apply the following symmetric and positive definite four-block 
preconditioner

$\left[\begin{array}{l|l|l|l|l}\mathbf{W} & & & \\ \hline & 1 & & & \\ & & 1 & & \\ \hline & & \mathbf{I}_{\mathbb{I}^{(\ell)}}^{\top} \mathbf{V I}_{\mathbb{I}^{(\ell)}} & \\ \hline & & & \mathbf{I}_{\mathbb{I}^{(\ell)}}^{\top} \mathbf{V I}_{\overline{\mathbb{I}}^{(\ell)}}\end{array}\right]$,

where $\underline{\mathbb{I}}^{(\ell)}=\underline{I}_{1}^{(\ell)} \cup \underline{I}_{2}^{(\ell)}, \overline{\mathbb{I}}^{(\ell)}=\overline{\mathbb{I}}_{1}^{(\ell)} \cup \overline{\mathbb{I}}_{2}^{(\ell)}$ and $\mathbf{W}$ is an approximation to the inverse $\mathbf{V}^{-1}$ of the system matrix $\mathbf{V}$. Since $\mathbf{V}$ is represented by an $\mathcal{H}$-matrix, we shall make use of the $\mathcal{H}$-matrix arithmetic (see $[13,14,15]$ ) to construct an appropriate approximation $\mathbf{W}$ to $\mathbf{V}^{-1}$. Namely, the $\mathcal{H}$-matrix $\mathbf{W}$ is computed with the help of the recursive block Gaussian elimination, as proposed in [14]. Recall that, in the present discretization based on a parametric surface, the underlying cluster tree is a balanced quad-tree which simplifies and speeds up the $\mathcal{H}$-matrix arithmetic, see [8] for details.

\section{Numerical RESUlts}

5.1. First example. In our first numerical example, we consider only one single manifold in $\mathbb{R}^{3}$; i.e., $n=3$ and $\Gamma=\Gamma_{1}$. We choose $\Gamma_{1}$ as the surface of the torus which is obtained by revolving the two-dimensional disk $B_{1}(3,0)=\left\{\mathbf{x} \in \mathbb{R}^{2}: \| \mathbf{x}-\right.$ $(3,0) \| \leq 1\}$ embedded in the $(x, y)$-plane in $\mathbb{R}^{3}$ about the $y$-axis in $\mathbb{R}^{3}$. This surface is represented by 9 four-sided patches. Furthermore, we apply no external field (i.e., $f \equiv 0)$ and set $g \equiv 1, h \equiv 1$, and $a_{1}=100$. For the numerical computations, we choose the level $j=5$ which yields a quadrangulation with about 37000 elements for the numerical approximation; i.e., the density $\lambda$ is approximated as a piecewise constant function with about 37000 degrees of freedom.

In case of $\alpha=2.9$, we observe that the charges are located at the outer most boundary of the torus. This holds for the unconstrained Gauss variational problem (left plot of Figure 5.4) as well as for the constrained Gauss variational problem (right plot of Figure 5.4). The maximum of the density is nearly 2 in case of the unconstrained Gauss variational problem. The constraint (2.4) enforces that this maximum is reduced to 1 in case of the constrained Gauss variational problem. For both problems, there is a rapid transition between the zero and the nonzero charges.

Our observations are in good agreement with theoretical results. According to [21], the support of the logarithmic equilibrium measure is contained in the part with positive curvature. The logarithmic kernel can be understood as a limiting case of the Riesz kernel as $\alpha \rightarrow 3$ from below. Thus, for $\alpha$ close to 3, one would expect a behavior similar to the logarithmic case. In fact, it is shown in [7] that there are 



FiguRE 5.4. The unconstrained density (left) and the constrained density (right) with respect to the torus in case of $\alpha=2.9$.

surfaces of revolution for which the support of the equilibrium measure is a proper subset of the outer boundary for $3-1 / 3<\alpha<3$.


FiguRE 5.5. The unconstrained density (left) and the constrained density (right) with respect to the torus in case of $\alpha=2$.

Next, we consider the case $\alpha=2$. Then, the boundary integral operator $V$ coincides with the traditional single layer operator for the Laplacian in $\mathbb{R}^{3}$. The numerical results are seen in Figure 5.5 where the left plot shows the solution of the unconstrained Gauss variational problem and the right plot shows the solution of the constrained Gauss variational problem. We observe a similar situation as for $\alpha=2.9$; i.e., the charges prefer to sit at the outer most boundary of the torus. Nevertheless, this effect is now not so extreme, in particular, the density is now everywhere positive. 
The maximum of the density is about 1.17 in case of the unconstrained Gauss variational problem and 1 in case of the constrained Gauss variational problem. Note that there is no rapid transition any more in case of the unconstrained Gauss variational problem.

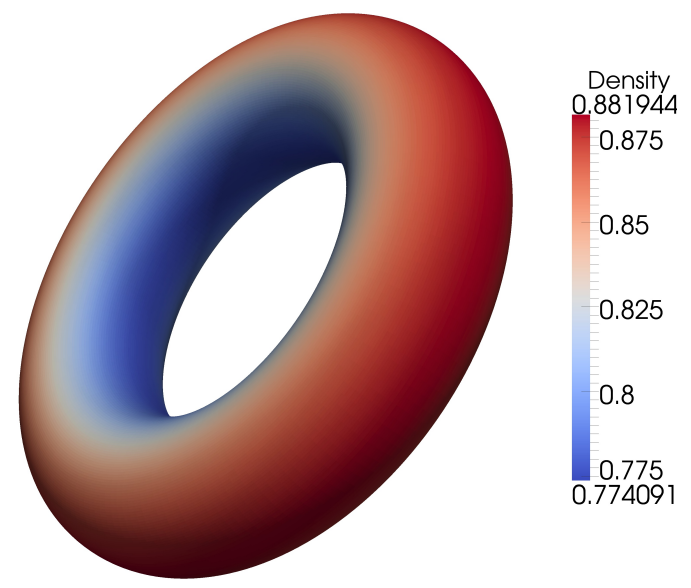

FiguRE 5.6. The density with respect to the torus in case of $\alpha=1.1$.

Finally, we choose $\alpha=1.1$. In this case, the solution of the unconstrained Gauss variational problem is nearly equally distributed over the whole torus, cf. Figure 5.6. In particular, the nonnegativity constraint never becomes active since the minimum of the charges is about 0.77 . Since also the maximum of the density is about 0.88 and thus less than 1, the solution of the unconstrained and constrained Gauss variational problem coincide. Again, our results validate theoretical findings. For $\alpha$ near 1, one would expect to have an (almost) uniform distribution according to the surface area measure (c.f. the Poppy-Seed Bagel Theorem), see e.g. [19, 20].

\begin{tabular}{|c|c|c|c|c|c|c|c|c|}
\hline & \multicolumn{7}{|c|}{ multilevel iteration } & \multirow{2}{*}{$\begin{array}{c}\text { single-level } \\
\text { iteration }\end{array}$} \\
\hline & level & $j=1$ & $j=2$ & $j=3$ & $j=4$ & $j=5$ & $j=6$ & \\
\hline$\alpha=1.1$ & $\begin{array}{c}\text { unconstrained } \\
\text { constrained }\end{array}$ & 1 & 1 & 1 & 1 & 1 & 1 & 1 \\
\hline \multirow{2}{*}{$\alpha=2.0$} & unconstrained & 1 & 1 & 1 & 1 & 1 & 1 & 1 \\
\hline & constrained & 2 & 1 & 2 & 1 & 2 & 1 & 7 \\
\hline \multirow{2}{*}{$\alpha=2.9$} & unconstrained & 2 & 1 & 2 & 1 & 2 & 1 & 25 \\
\hline & constrained & 4 & 7 & 4 & 7 & 3 & 19 & 59 \\
\hline
\end{tabular}

TABLE 5.1. The number of iterations which are required for solving the Gauss variational problem on the torus. 
We finally shall comment on the iterative solution of the discretized optimization problem by the primal-dual active set strategy. It is seen in Table 5.1 how many iterations per level are needed by the active set strategy when using the multilevel strategy which is proposed in Subsection 4.2. Since all inequality constraints are inactive if $\alpha=1.1$ for the unconstrained and constrained Gauss variational problem and if $\alpha=2.0$ for the unconstrained Gauss variational problem, the solution is always found in the first iteration since the active sets are empty. This of course holds also true if no multilevel iteration is used, see the last column of Table 5.1. However, if $\alpha=2.0$ for the unconstrained Gauss variational problem and if $\alpha=2.9$ for the unconstrained and constrained Gauss variational problem, a lot of iterations are saved by employing the multilevel iteration.

5.2. Second example. In our second example, we consider $\Gamma_{1}$ to be the surface of the drilled cube

$$
\Omega_{1}=\left\{\left(x_{1}, x_{2}, x_{3}\right) \in(-1,1)^{3}: x_{1}^{2}+x_{2}^{2}>\frac{1}{4} \wedge x_{2}^{2}+x_{3}^{2}>\frac{1}{4} \wedge x_{3}^{2}+x_{1}^{2}>\frac{1}{4}\right\}
$$

which is is represented by 48 four-sided patches. The manifold $\Gamma_{2}$ is the surface of the domain $\Omega_{2}$ which is given as the union of the following three barbells

$$
\begin{aligned}
\Omega_{2} & =\left\{\left(x_{1}, x_{2}, x_{3}\right) \in\left(-\frac{5}{2}, \frac{5}{2}\right)^{3}: x_{1}^{2}+x_{2}^{2}<\frac{1}{16} \vee x_{2}^{2}+x_{3}^{2}<\frac{1}{16}^{2} \vee x_{3}^{2}+x_{1}^{2}<\frac{1}{16}\right\} \\
& \cup\left\{B_{1}\left(m_{1}, m_{2}, m_{3}\right): m_{1}, m_{2}, m_{3}= \pm \frac{5}{2}\right\} .
\end{aligned}
$$

It is represented by 54 four-sided patches. Both domains $\Omega_{1}$ and $\Omega_{2}$ are entangled as seen in the subsequent figures of this subsection. Likewise to the first example, we apply no external field (i.e., $f \equiv 0$ ) and choose $g_{i} \equiv 1, h_{i} \equiv 10$, and $a_{i}=100$ for $i=1,2$. The densities $\lambda_{1}$ and $\lambda_{2}$ are approximated on level 5 which corresponds to about 105000 piecewise constants boundary elements.

For the unconstrained Gauss variational problem in case of $\alpha=2.9$, we obtain the density which is presented in Figure 5.7. To improve visualization this figure and all the subsequent figures show the modulus of the charges even though they are of opposite sign on the drilled cube and the three barbells. The maximum of the densities is about 80 and assumed on the drilled cube. It is clearly seen in the clipped plot on the right hand side of Figure 5.7 that the charges are located at the edges of the drilled cube and the poles of the barbells. This effect is enhanced if we constrain the charges to be less than 10, as seen in Figure 5.8. Like in the first example, we observe a rapid transition between the zero and the nonzero charges.

If the kernel parameter $\alpha$ is set to $\alpha=2$, the density of the unconstrained Gauss variational problem is everywhere positive as seen in Figure 5.9. The maximum of 

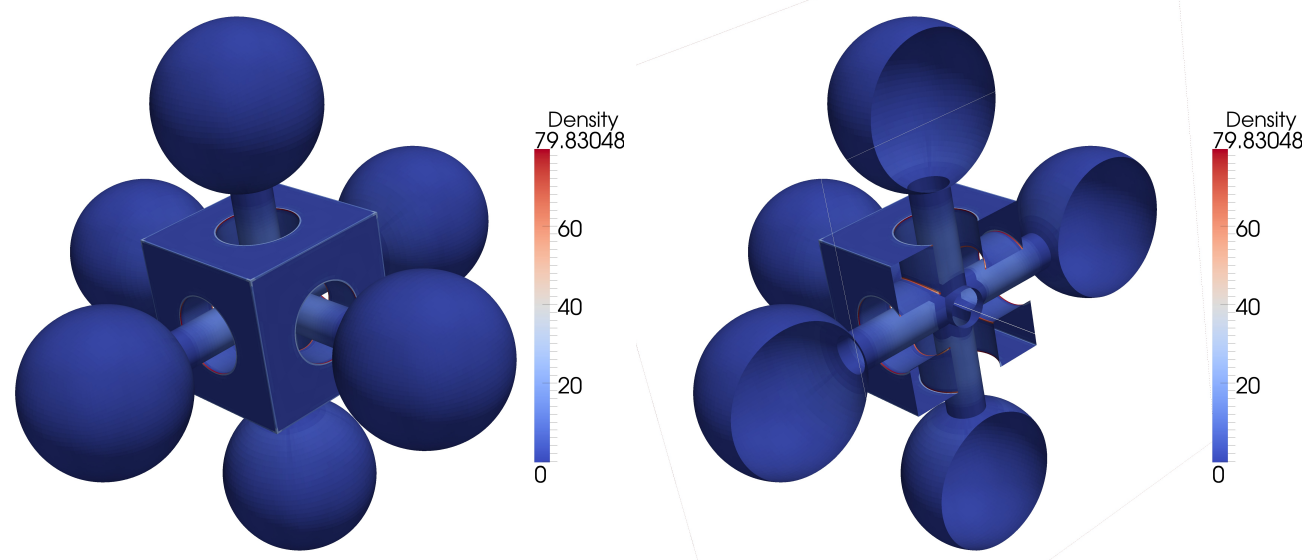

FiguRE 5.7. Solution for the second example in case of the unconstrained Gauss variational problem and $\alpha=2.9$.


Figure 5.8. Solution for the second example in case of the constrained Gauss variational problem and $\alpha=2.9$.

the densities is about 13 and assumed at the vertices and the interior edges of the drilled cube, as seen in the clipped plot on the right hand side of Figure 5.9. The maximum charges of the three barbells are again located on the poles of the barbells. If we constrain the charges to be less than 10, we get the densities found in Figure 5.10 where the mentioned effects are again enhanced.

Finally, for $\alpha=1.1$, we make nearly the same observations as in the corresponding case of the first example. All charges are positive everywhere and nearly equally distributed relative to the surfaces $\Gamma_{1}$ and $\Gamma_{2}$, see Figure 5.11. The minimum charge on the three barbells is about 0.85 and the maximum charge is about 2.5. The minimum charge on the drilled cube is about 3.0 and the maximum charge is about 4.0. Hence, neither the lower constraint 0 nor the upper constrain 10 are ever active. 



FiguRE 5.9. Solution for the second example in case of the unconstrained Gauss variational problem and $\alpha=2$.
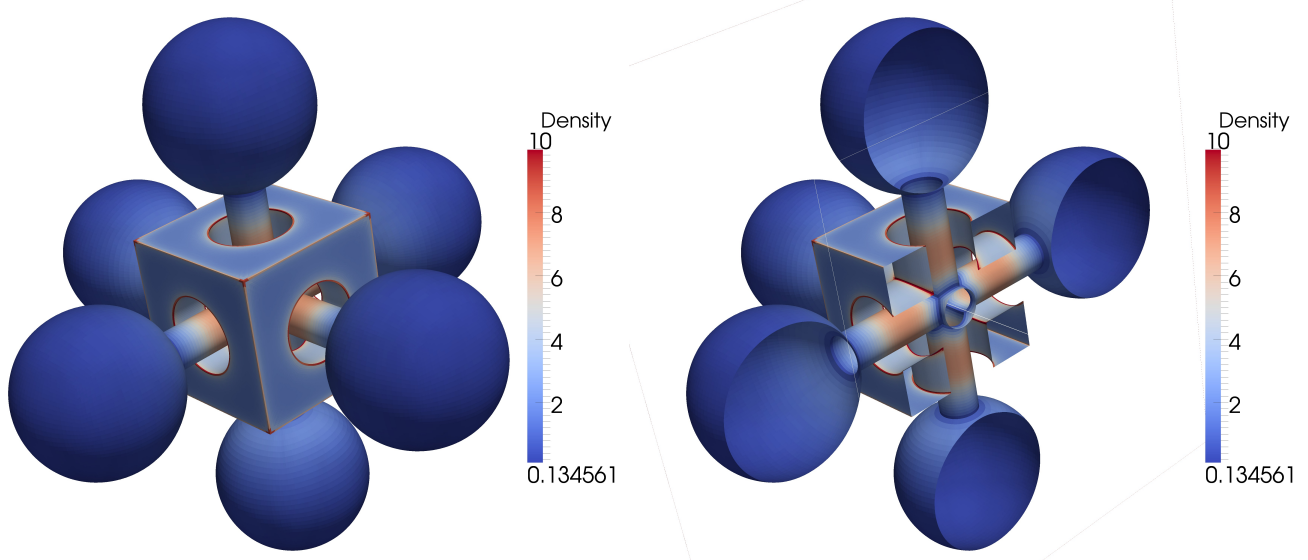

FiguRE 5.10. Solution for the second example in case of the constrained Gauss variational problem and $\alpha=2$.

In particular, the solutions of the constrained and unconstrained Gauss variational problem coincide.

The iterations per level which are needed by the multilevel version of the primaldual active set strategy are found in Table 5.2. In comparison with the single-level iterations, we save some of the iterations on the finest level. Nevertheless, the effect is not so strong as in case of the torus example since there are not very many iterations needed at all.

\section{Conclusion}

In the present article, we have demonstrated that the primal-dual active set strategy provides in combination with an $\mathcal{H}$-matrix based fast boundary element method an 

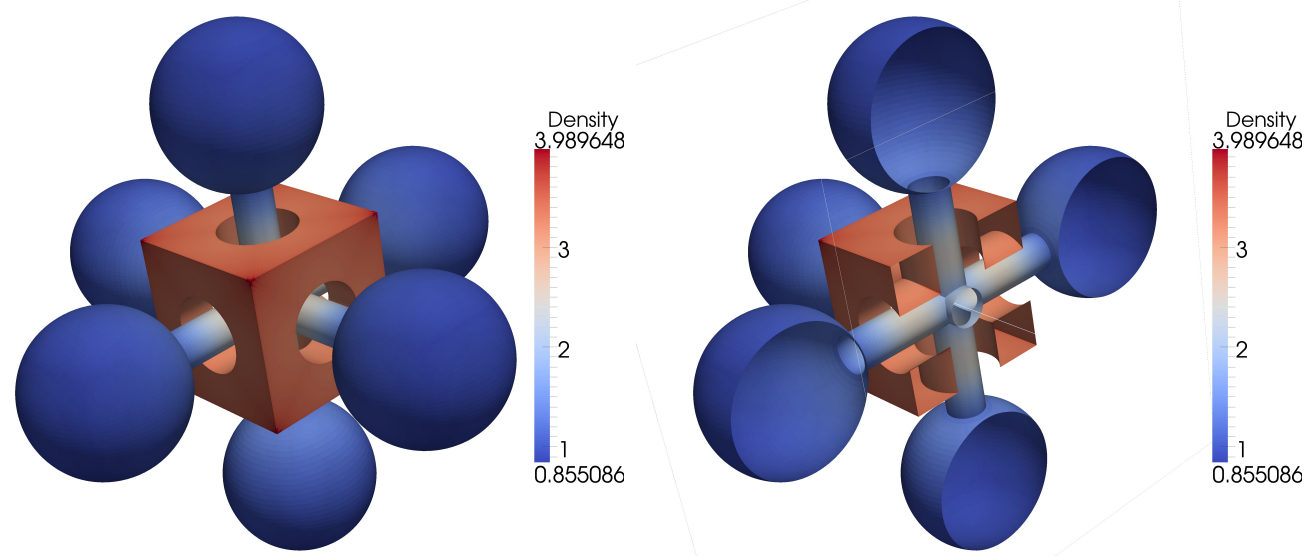

Figure 5.11. Solution for the second example in case of the Gauss variational problem and $\alpha=1.1$.

\begin{tabular}{|c|c|c|c|c|c|c|c|}
\cline { 2 - 8 } \multicolumn{1}{c|}{} & \multicolumn{5}{c|}{ multilevel iteration } & single-level \\
\cline { 2 - 8 } \multicolumn{1}{c|}{} & level & $j=1$ & $j=2$ & $j=3$ & $j=4$ & $j=5$ & iteration \\
\hline$\alpha=1.1$ & $\begin{array}{c}\text { unconstrained } \\
\text { constrained }\end{array}$ & 1 & 1 & 1 & 1 & 1 & 1 \\
\hline \multirow{2}{*}{$\alpha=2.0$} & unconstrained & 1 & 1 & 1 & 1 & 1 & 1 \\
& constrained & 1 & 1 & 1 & 2 & 2 & 2 \\
\hline \multirow{2}{*}{$\alpha=2.9$} & unconstrained & 2 & 4 & 4 & 5 & 7 & 11 \\
& constrained & 2 & 5 & 7 & 6 & 10 & 17 \\
\hline
\end{tabular}

TABLE 5.2. The number of iterations which are required for solving the Gauss variational problem on the drilled cube and the three barbells.

efficient approach to computationally solve constrained and unconstrained Riesz minimal energy problems. We followed hereby the paradigm "first discretize then optimize".

Another approach to solve Riesz minimal energy problems would be "first optimize then discretize". This would mean to introduce Lagrange multipliers for the inequality constraints of the continuous optimization problem. Since the densities lie in $H^{-\varepsilon / 2}\left(\Gamma_{1} \cup \Gamma_{2}\right)$, the Lagrange multipliers need to belong to $H^{\varepsilon / 2}\left(\Gamma_{1} \cup \Gamma_{2}\right)$. In case of $\varepsilon=\alpha-1>1$, the latter cannot be discontinuous functions. Hence, globally continuous piecewise linear boundary elements would be necessary to discretize the Lagrange multipliers of this optimization problem. 


\section{REFERENCES}

[1] R. Backofen, M. Gräf, D. Potts, S. Praetorius, A. Voigt, and T. Witkowski. A continuous approach to discrete ordering on $\mathbb{S}^{2}$. SIAM J. Multiscale Model. Simul. 9 (2011), 314-334.

[2] J.S. Brauchart, P.D. Dragnev, and E.B. Saff. An electrostatic problem on the sphere arising from a nearby point charge. In K. Ivanov et al., editors, Constructive Theory of Functions, pages 1-45, Prof. Marin Drinov Academic Publishing House, Sofia (2014).

[3] M. Bebendorf. Approximation of boundary element matrices. Numer. Math. 86 (2000), 565-589.

[4] M. Bebendorf and S. Rjasanow. Adaptive low-rank approximation of collocation matrices. Computing $\mathbf{7 0}$ (2003), 1-24.

[5] M. Benzi and A.J. Wathen. Some preconditioning techniques for saddle point problems. In W.H.A. Schilders et al., editors, Model Order Reduction: Theory, Research Aspects and Applications, volume 13 of Mathematics in Industry, pages 195-211, Springer, Heidelberg (2008).

[6] M. Bergounioux, K. Ito, and K. Kunisch. Primal-dual strategy for constrained optimal control problems. SIAM J. Contr. Optim. 37 (1999), 1176-1194.

[7] J. Brauchart, D.P. Hardin, and E.B. Saff. The support of the limit distribution of optimal Riesz energy points on sets of revolution in $\mathbb{R}^{3}$. J. Math. Phys. 48 (2007), 122901.

[8] J. Dölz, H. Harbrecht, and M. Peters. $\mathcal{H}$-matrix accelerated second moment analysis for potentials with rough correlation. J. Sci. Comput. 65 (2015), 387-410.

[9] P.D. Dragnev and E.B. Saff. Constrained energy problems with applications to orthogonal polynomials of a discrete variable. J. Anal. Math. 72 (1997), 223-259.

[10] C.F. Gauß. Allgemeine Lehrsätze in Beziehung auf die im verkehrten Verhältnisse des Quadrats der Entfernung wirkenden Anziehungs- und Abstoßungs-Kräfte (1839). Werke 5 (1867), 197-244.

[11] G.H. Golub and C.F. Van Loan. Matrix Computations. Fourth edn., Johns Hopkins University Press, Baltimore, 2012.

[12] L. Greengard and V. Rokhlin. A fast algorithm for particle simulation. J. Comput. Phys. 73 (1987), 325-348.

[13] W. Hackbusch. A sparse matrix arithmetic based on $\mathcal{H}$-matrices. Part I: Introduction to $\mathcal{H}$-matrices. Computing 62 (1999), 89-108.

[14] W. Hackbusch. Hierarchische Matrizen: Algorithmen und Analysis. Springer, BerlinHeidelberg, 2009.

[15] W. Hackbusch and B.N. Khoromskij. A sparse $\mathcal{H}$-matrix arithmetic. General complexity estimates. J. Comput. Appl. Math. 125 (2000), 479-501.

[16] H. Harbrecht and M. Peters. Comparison of fast boundary element methods on parametric surfaces. Comput. Methods Appl. Mech. Engrg. 261-262 (2013), 39-55.

[17] H. Harbrecht, W.L. Wendland, and N. Zorii. On Riesz minimal energy problems. J. Math. Anal. Appl. 393 (2012), 397-412. 
[18] H. Harbrecht, W.L. Wendland, and N. Zorii. Riesz minimal energy problems on $C^{k-1,1}$-manifolds. Math. Nachr. 287 (2014) 48-69.

[19] D.P. Hardin and E.B. Saff. Discretizing manifolds via minimum energy points. Notices of the AMS $\mathbf{5 1}$ (2004), 1186-1194.

[20] D.P. Hardin and E.B. Saff. Minimal Riesz energy point configurations for rectifiable d-dimensional manifolds. Advances in Math. 193 (2004), 174-204.

[21] D.P. Hardin, E.B. Saff, and H. Stahl. The support of the logarithmic equilibrium measure on sets of revolution in $\mathbb{R}^{3}$. J. Math. Phys. 48 (2007), 022901.

[22] M. Hintermüller, K. Ito, and K. Kunisch. The primal-dual active set strategy as a semismooth Newton method. SIAM J. Optim. 13 (2003), 865-888.

[23] J. Hoschek and D. Lasser. Grundlagen der geometrischen Datenverarbeitung. Teubner, Stuttgart, 1989.

[24] K. Ito and K. Kunisch. The primal-dual active set method for nonlinear optimal control problems with bilateral constraints. SIAM J. Contr. Optim. 43 (2004), 357376.

[25] W. Karush. Minima of Functions of Several Variables with Inequalities as Side Constraints. M.Sc. Dissertation, Dept. of Mathematics, University of Chicago, Chicago, Illinois, 1939.

[26] S.O. Kasap. Principles of Electrical Engineering Materials and Devices. Erwin McGraw Hill, Boston (1997).

[27] H.W. Kuhn and A.W. Tucker. Nonlinear programming. In Proceedings of 2nd Berkeley Symposium, pages 481-492, University of California Press, Berkeley (1951).

[28] K. Kunisch and A. Rösch. Primal-dual active set strategy for a general class of constrained optimal control problems. SIAM J. Optim. 13 (2002), 321-334.

[29] N.S. Landkof. Foundations of Modern Potential Theory. Springer, Berlin (1972).

[30] G. Of, W.L. Wendland, and N. Zorii. On the numerical solution of minimal energy problems. Complex Var. Elliptic Equ. 55 (2012), 991-1012.

[31] M. Ohtsuka. On potentials in locally compact spaces. J. Sci. Hiroshima Univ. Ser. A1 25 (1961), 135-352.

[32] I. Perugia, V. Simoncini, and M. Arioli. Linear algebra methods in a mixed approximation of magnetostatic problems. SIAM J. Sci. Comput. 21 (1999), 1085-1101.

[33] E.A. Rakhmanov. Equilibrium measure and the distribution of zeros of extremal polynomials of a discrete variable. Sb. Math. 187 (1996), 1213-1228.

[34] E.B. Saff and V. Totik. Logarithmic Potentials with External Fields. Springer, Berlin (1997).

[35] D. Silvester and A. Wathen. Fast iterative solution of stabilised Stokes systems part II: Using general block preconditioners. SIAM J. Numer. Anal. 31 (1994), 1352-1367.

[36] J.J. Thomson. On the structure of the atom: An investigation of the stability and periods of oscillation of a number of corpuscles arranged at equal intervals around the circumference of a circle; with application of the results on the theory of atomic structure. Philos. Mag. 7 (1904), 237-265. 
[37] N. Zorii. On the solvability of the Gauss variational problem. Comp. Methods Function Theory 2 (2002), 427-448.

[38] N. Zorii. Equilibrium potentials with external fields. Ukrain. Math. J. 55 (2003), 1423-1444.

[39] N. Zorii. Equilibrium problems for potentials with external fields. Ukrain. Math. J. 55 (2003), 1588-1618.

[40] N. Zorii. On the constrained Gauss variational problem. Bull. Soc. Sci. Lettr. Eódź 53 (2003), 25-40.

[41] N. Zorii. Necessary and sufficient conditions for the solvability of the Gauss variational problem. Ukrain. Math. J. 57 (2005), 70-99.

[42] N. Zorii. Constrained energy problems with external fields. Complex Anal. Oper. Theory 5 (2011), 775-785.

[43] N. Zorii. Constrained energy problems with external fields for vector measures. Math. Nachr. 285 (2012), 1144-1165.

[44] N. Zorii. Equilibrium problems for infinite dimensional vector potentials with external fields. Potential Anal. 38 (2013), 397-432.

[45] W. Zulehner. Analysis of iterative methods for saddle point problems: a unified approach. Math. Comput. 71 (2002), 479-505.

Helmut Harbrecht, Universität Basel, Departement Mathematik und Informatik, Spiegelgasse 1, 4051 Basel, Switzerland

E-mail address: helmut.harbrecht@unibas.ch

Wolfgang L. Wendland, Universität Stuttgart, Institut für Angewandte Analysis und Numerische Simulation, Pfaffenwaldring 57, 70569 Stuttgart, GERMANY

E-mail address: wendland@mathematik.uni-stuttgart.de

Natalia Zorit, Institute of Mathematics, National Academy of Sciences of Ukraine, Tereshchenkivska 3, 01601, Kyiv-4, Ukraine

E-mail address: natalia.zorii@gmail.com 